\title{
Supporting material for: autoBioSeqpy: a deep learning tool for the classification of biological sequences
}

\author{
Runyu Jing ${ }^{1}$, Yizhou Li ${ }^{1}$, Li Xue ${ }^{2}$, Fengjuan Liu ${ }^{3}$, Menglong $\mathrm{Li}^{4^{*}}$ and Jiesi Luo ${ }^{5^{*}}$ \\ ${ }^{1}$ College of Cybersecurity, Sichuan University, Chengdu, China, 610065 \\ ${ }^{2}$ School of Public Health, Southwest Medical University, Luzhou, Sichuan, China, \\ 646000 \\ ${ }^{3}$ School of Geography and Resources, Guizhou Education University, Guiyang, China, \\ 550018 \\ ${ }^{4}$ College of Chemistry, Sichuan University, Chengdu, China, 610065 \\ ${ }^{5}$ Department of Pharmacology, School of Pharmacy, Southwest Medical University, \\ Luzhou, Sichuan, China, 646000 \\ * To whom correspondence should be addressed. \\ Jiesi Luo
}

Email address: 1js@swmu.edu.cn

Menglong Li

Email address: liml@scu.edu.cn 


\begin{tabular}{cc}
\hline Metric & Calculation \\
\hline Precision (PRE) & $P R E=\frac{T P}{T P+F P}$ \\
Accuracy (ACC) & $A C C=\frac{T P+T N}{T P+F P+T N+F N}$ \\
$F$-value & $F-$ value $=2 \times \frac{T P}{2 T P+F P+F N}$ \\
Recall & Recall $=\frac{T P}{T P+F N}$ \\
Matthew's correlation coefficient (MCC) & $(T P \times T N)-(F N \times F P)$ \\
Receiver Operating Characteristic (ROC) curve & Plot the true positive rate against the false positive rate at \\
Area Under the ROC Curve (AUC) & various thresholds \\
Precision-Recall (PR) curve & The area under the ROC curve
\end{tabular}

Where $T P, T N, F P$ and $F N$ represent the numbers of true positives, true negatives, false positives and false negatives, respectively 
Table S2. Detailed description of five deep learning architectures related to T3SS effectors prediction

\begin{tabular}{|c|c|c|}
\hline Layer (type) & Output Shape & Param \\
\hline \multicolumn{3}{|c|}{ Long Short-Term Memory neural network (LSTM) } \\
\hline embedding_1 (Embedding) & (None, None, 256) & 6656 \\
\hline 1stm_1 (LSTM) & (None, 64) & 82176 \\
\hline dense_1 (Dense) & (None, 1$)$ & 65 \\
\hline activation_1 (Activation) & (None, 1$)$ & 0 \\
\hline \multicolumn{3}{|c|}{ Bidirectional Long Short-Term Memory neural network (biLSTM) } \\
\hline embedding_1 (Embedding) & (None, None, 256) & 6656 \\
\hline bidirectional_1 (Bidirectional LSTM) & (None, 128) & 164352 \\
\hline dropout_1 (Dropout) & (None, 128) & 0 \\
\hline dense_1 (Dense) & (None, 1$)$ & 129 \\
\hline activation_1 (Activation) & (None, 1$)$ & 0 \\
\hline \multicolumn{3}{|c|}{ Convolutional neural network (CNN) } \\
\hline embedding_1 (Embedding) & (None, 100, 256) & 6656 \\
\hline dropout_1 (Dropout) & (None, 100, 256) & 0 \\
\hline conv1d_1 (Conv1D) & (None, 96,250 ) & 320250 \\
\hline max_pooling1d_1 (MaxPooling) & (None, 48,250 ) & 0 \\
\hline conv1d_2(Conv1D) & (None, 44,250$)$ & 312750 \\
\hline max_pooling1d_2 (MaxPooling) & (None, 22,250$)$ & 0 \\
\hline dropout_2 (Dropout) & (None, 22, 250) & 0 \\
\hline flatten_1 (Flatten) & (None, 5500) & 0 \\
\hline dense_1 (Dense) & (None, 650$)$ & 3575650 \\
\hline dropout_3 (Dropout) & (None, 650$)$ & 0 \\
\hline activation_1 (Activation) & (None, 650) & 0 \\
\hline dense_2 (Dense) & (None, 1) & 651 \\
\hline activation 2 (Activation) & (None, 1) & 0 \\
\hline
\end{tabular}

Architecture consisting of Convolutional neural network and Long Short-term memory neural network (CNNLSTM)

\begin{tabular}{ccc}
\hline embedding_1 (Embedding) & (None, None, 256) & 6656 \\
dropout_1 (Dropout) & (None, None, 256) & 0 \\
conv1d_1 (Conv1D) & (None, None, 250) & 320250 \\
max_pooling1d_1 (MaxPooling) & (None, None, 250) & 0 \\
lstm_1 (LSTM) & (None, 64) & 80640 \\
dense_1 (Dense) & (None, 1) & 65 \\
activation_1 (Activation) & (None, 1) & 0 \\
\hline
\end{tabular}

Architecture consisting of Convolutional neural network and Bidirectional Long Short-term memory neural network (CNN-biLSTM)

\begin{tabular}{ccc}
\hline embedding_1 (Embedding) & (None, None, 256) & 6656 \\
dropout_1 (Dropout) & (None, None, 256) & 0 \\
conv1d_1 (Conv1D) & (None, None, 250) & 320250 \\
max_pooling1d_1 (MaxPooling) & (None, None, 250) & 0 \\
conv1d_2 (Conv1D) & (None, None, 250) & 312750
\end{tabular}


max_pooling1d 2 (MaxPooling) conv1d_3 (Conv1D)

max_pooling1d_3 (MaxPooling) conv1d 4 (Conv1D)

max_pooling1d_4 (MaxPooling)

bidirectional_1 (Bidirectional LSTM)

dense_1 (Dense)

activation_1(Activation)
(None, None, 250)

(None, None, 250)

(None, None, 250)

(None, None, 250)

(None, None, 250)

(None, 128)

(None, 1)

(None, 1)
0

312750

0

312750

0

161280

129 
Table S3. Detailed description of deep learning architectures related to CRISPR sgRNA efficiency prediction

\begin{tabular}{|c|c|c|}
\hline Layer (type) & Output Shape & Param \\
\hline \multicolumn{3}{|c|}{ Convolutional neural network with $k$-mer $(k=$ not used, 2,3$)$ dictionary encoding } \\
\hline embedding_1 (Embedding) & $\begin{array}{c}\text { (None, } 30,16),(\text { None, } 29,64), \text { (None, } \\
28,256)\end{array}$ & $80,1280,25600$ \\
\hline conv1d_1 (Conv1D) & $\begin{array}{c}\text { (None, 26, 80), (None, 25, 80), (None, } \\
24,80)\end{array}$ & $6480,25680,102480$ \\
\hline average_pooling1d_1 (Average Pooling) & $\begin{array}{c}\text { (None, } 13,80),(\text { None, } 12,80), \text { (None, } \\
12,80)\end{array}$ & 0 \\
\hline flatten_1 (Flatten) & (None, 1040), (None, 960), (None, 960) & 0 \\
\hline dropout_1 (Dropout) & (None, 1040), (None, 960), (None, 960) & 0 \\
\hline dense_1 (Dense) & (None, 80 ) & $83280,76880,76880$ \\
\hline dropout_2 (Dropout) & (None, 80 ) & 0 \\
\hline dense_2 (Dense) & (None, 40 ) & 3240 \\
\hline dropout_3 (Dropout) & (None, 40) & 0 \\
\hline dense_3 (Dense) & (None, 40$)$ & 1640 \\
\hline dropout_4 (Dropout) & (None, 40) & 0 \\
\hline activation_1 (Activation) & (None, 40 ) & 0 \\
\hline dense_4 (Dense) & (None, 1$)$ & 41 \\
\hline activation_2 (Activation) & (None, 1 ) & 0 \\
\hline \multicolumn{3}{|c|}{ Convolutional neural network with $k$-mer $(k=$ not used, 2,3$)$ one-hot encoding } \\
\hline conv2d_1 (Conv2D) & $\begin{array}{c}\text { (None, } 1,26,80),(\text { None }, 1,25,80) \\
(\text { None, } 1,24,80)\end{array}$ & $1680,6480,25680$ \\
\hline average_pooling2d_1 (Average Pooling) & $\begin{array}{c}\text { (None, } 1,13,80),(\text { None, } 1,12,80) \\
(\text { None }, 1,12,80)\end{array}$ & 0 \\
\hline flatten_1 (Flatten) & (None, 1040), (None, 960), (None, 960) & 0 \\
\hline dropout_1 (Dropout) & (None, 1040), (None, 960), (None, 960) & 0 \\
\hline dense_1 (Dense) & (None, 80$)$ & $83280,76880,76880$ \\
\hline dropout_2 (Dropout) & (None, 80$)$ & 0 \\
\hline dense_2 (Dense) & (None, 40 ) & 3240 \\
\hline dropout_3 (Dropout) & (None, 40) & 0 \\
\hline dense_3 (Dense) & (None, 40 ) & 1640 \\
\hline dropout_4 (Dropout) & (None, 40 ) & 0 \\
\hline dense_4 (Dense) & (None, 1 ) & 41 \\
\hline activation_1 (Activation) & (None, 1 ) & 0 \\
\hline
\end{tabular}


Table S4. Number of proteins in each location and sub-locations

\begin{tabular}{lll}
\hline Class Name & Training & Test \\
\hline Cell membrane & 1067 & 273 \\
Cytoplasm & 2180 & 508 \\
Endoplasmic reticulum & 689 & 173 \\
Golgi apparatus & 286 & 70 \\
Lysosome/Vacuole & 257 & 64 \\
Mitochondrion & 1208 & 302 \\
Nucleus & 3235 & 808 \\
Peroxisome & 124 & 30 \\
Plastid & 605 & 152 \\
Extracellular & 1580 & 393 \\
\hline
\end{tabular}


Table S5. Detailed description of the deep learning architecture related to subcellular localization classification

\begin{tabular}{ccc}
\hline Layer (type) & Output Shape & Param \\
\hline embedding_1 (Embedding) & (None, 2000, 256) & 6656 \\
dropout_1 (Dropout) & (None, 2000, 256) & 0 \\
conv1d_1 (Conv1D) & (None, 1996, 250) & 320250 \\
max _pooling1d_1 (Max Pooling) & $($ None, 998, 250) & 0 \\
bidirectional_1 (Bidirection LSTM) & $($ None, 998, 128) & 161280 \\
seq_self_attention_1 (Self Attention) & $($ None, 998, 128) & 8257 \\
flatten_1 (Flatten) & (None, 127744) & 0 \\
dense_1 (Dense) & $($ None, 10) & 1277450 \\
activation_1 (Activation) & (None, 10) & 0 \\
\hline
\end{tabular}


Table S6. Performance comparison of different number of convolutional and pooling layers on the 10-time test dataset

\begin{tabular}{cccccc}
\hline No. of Conv and pooling layers & ACC (\%) & F-value (\%) & Recall (\%) & PRE (\%) & $M C C$ \\
\hline 1 & $89.7 \pm 1.2$ & $83.6 \pm 1.6$ & $78.0 \pm 2.1$ & $90.2 \pm 3.9$ & $0.767 \pm 0.027$ \\
2 & $90.8 \pm 1.6$ & $85.9 \pm 2.1$ & $83.3 \pm 3.1$ & $89.0 \pm 5.1$ & $0.794 \pm 0.034$ \\
3 & $89.6 \pm 1.5$ & $84.4 \pm 1.9$ & $83.6 \pm 4.4$ & $85.8 \pm 5.4$ & $0.769 \pm 0.029$ \\
4 & $87.2 \pm 2.0$ & $80.5 \pm 3.0$ & $79.1 \pm 7.3$ & $83.0 \pm 6.4$ & $0.716 \pm 0.038$ \\
& & CNN-biLSTM & & & \\
1 & $91.4 \pm 1.1$ & $86.3 \pm 1.7$ & $81.7 \pm 2.5$ & $91.6 \pm 2.4$ & $0.804 \pm 0.025$ \\
3 & $91.7 \pm 1.1$ & $86.9 \pm 2.1$ & $82.2 \pm 4.4$ & $92.3 \pm 2.2$ & $0.812 \pm 0.025$ \\
4 & $91.4 \pm 1.2$ & $86.8 \pm 1.6$ & $83.7 \pm 4.5$ & $90.7 \pm 5.1$ & $0.809 \pm 0.025$ \\
& $91.8 \pm 1.3$ & $86.7 \pm 2.6$ & $80.9 \pm 6.0$ & $93.9 \pm 3.7$ & $0.815 \pm 0.030$ \\
2 & $83.1 \pm 3.7$ & $70.9 \pm 9.2$ & $63.8 \pm 13.9$ & $83.1 \pm 8.0$ & $0.613 \pm 0.088$ \\
3 & $80.2 \pm 7.4$ & $64.3 \pm 16.9$ & $56.8 \pm 19.4$ & $81.2 \pm 14.1$ & $0.547 \pm 0.173$ \\
4 & $78.8 \pm 5.8$ & $63.8 \pm 14.4$ & $60.5 \pm 23.2$ & $76.3 \pm 14.0$ & $0.530 \pm 0.137$ \\
\hline & $81.3 \pm 5.0$ & $68.3 \pm 10.3$ & $61.7 \pm 13.4$ & $78.6 \pm 9.8$ & $0.568 \pm 0.123$ \\
\hline
\end{tabular}


Table S7. Performance comparison of different model architectures using $k$-mer dictionary encoding on the 10time test dataset

\begin{tabular}{ccccccc}
\hline$k$-mer & Architecture & ACC $(\%)$ & $F$-value (\%) & Recall (\%) & PRE (\%) & $M C C$ \\
\hline Not used & \multirow{2}{*}{ biLSTM } & $85.9 \pm 1.5$ & $79.4 \pm 2.1$ & $81.4 \pm 2.7$ & $77.6 \pm 3.1$ & $0.688 \pm 0.032$ \\
2 & & $84.6 \pm 1.0$ & $72.7 \pm 2.3$ & $61.6 \pm 3.8$ & $89.2 \pm 3.6$ & $0.647 \pm 0.025$ \\
3 & & & & & \\
& & & & & & \\
Not used & & & & & & \\
2 & \multirow{2}{*}{ CNN-biLSTM } & $85.4 \pm 1.8 \pm 1.3$ & $86.7 \pm 2.6$ & $80.9 \pm 6.0$ & $93.9 \pm 3.7$ & $0.815 \pm 0.030$ \\
3 & & $83.0 \pm 1.0$ & $70.1 \pm 2.2$ & $59.5 \pm 3.3$ & $85.6 \pm 2.9$ & $0.607 \pm 0.024$ \\
\hline
\end{tabular}


Table S8. Performance comparison of different input sequence lengths on the 10-time test dataset

\begin{tabular}{ccccccc}
\hline Length & Architecture & ACC (\%) & $F$-value (\%) & Recall (\%) & PRE (\%) & MCC \\
\hline 100 & & $91.4 \pm 0.8$ & $86.4 \pm 1.7$ & $81.2 \pm 4.9$ & $92.6 \pm 3.1$ & $0.807 \pm 0.018$ \\
1000 & biLSTM & $91.0 \pm 2.2$ & $85.8 \pm 3.9$ & $81.6 \pm 6.5$ & $91.0 \pm 3.9$ & $0.798 \pm 0.050$ \\
2000 & & $90.6 \pm 1.5$ & $85.2 \pm 2.4$ & $80.8 \pm 3.5$ & $90.2 \pm 4.0$ & $0.786 \pm 0.035$ \\
& & & & & \\
100 & & & & & \\
1000 & \multirow{2}{*}{ CNN-biLSTM } & $81.8 \pm 1.3$ & $86.7 \pm 2.6$ & $80.9 \pm 6.0$ & $93.9 \pm 3.7$ & $0.815 \pm 0.030$ \\
2000 & & $89.5 \pm 1.0$ & $83.3 \pm 2.0$ & $78.8 \pm 5.2$ & $88.8 \pm 3.5$ & $0.761 \pm 0.023$ \\
\hline
\end{tabular}




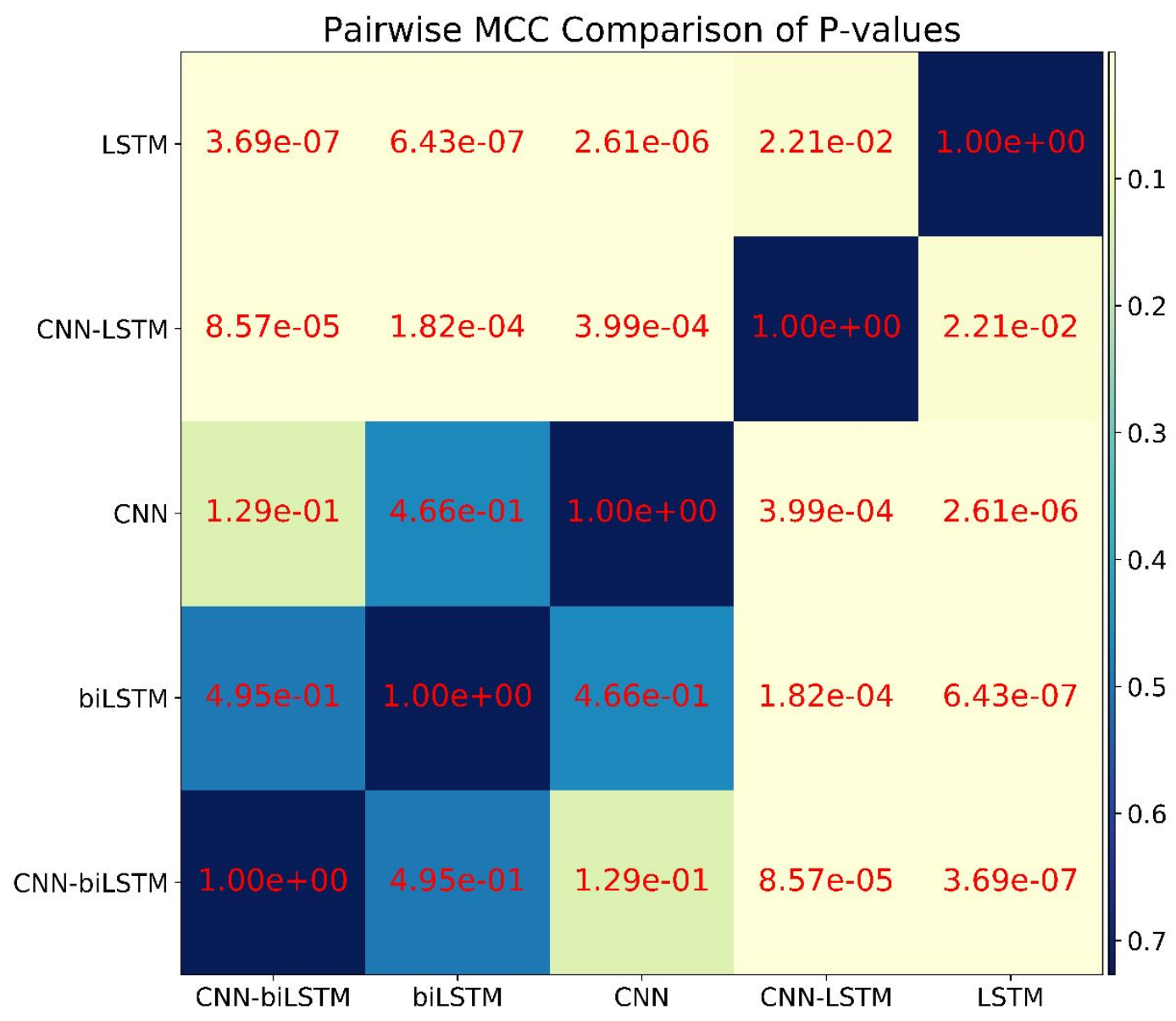

Figure S1. The $p$-values of pairwise model comparison are calculated by using the Wilcoxon signed-rank test. 


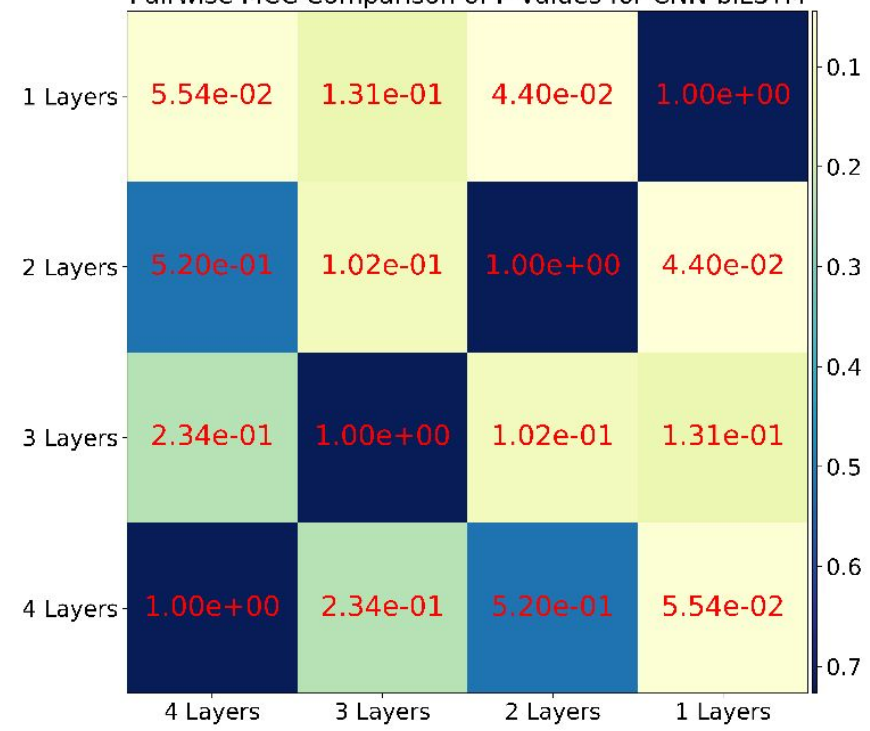

Pairwise MCC Comparison of P-values for CNN

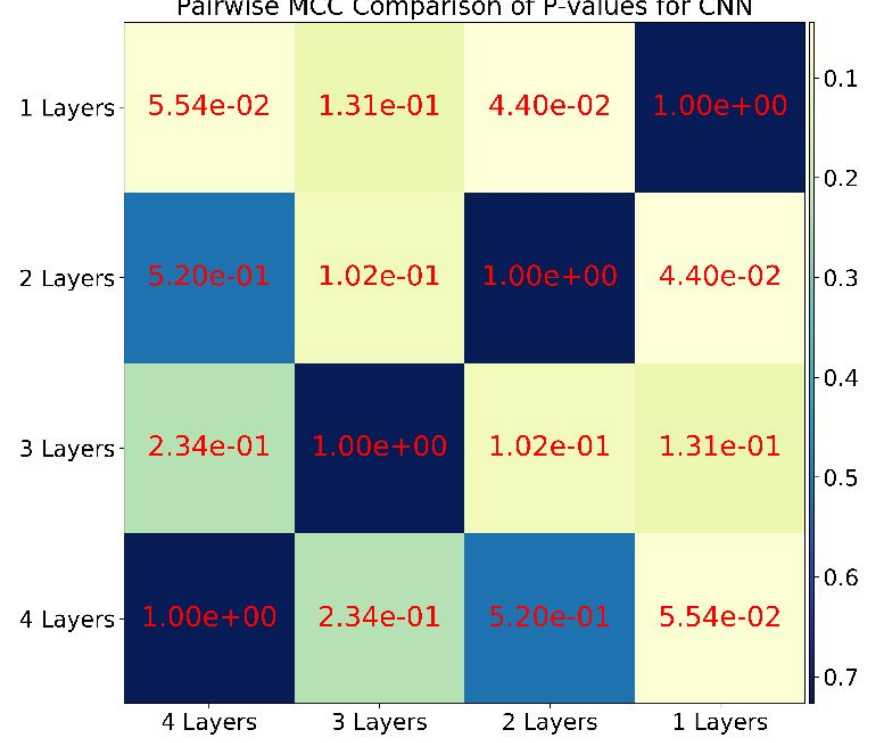

Pairwise MCC Comparison of P-values for CNN-LSTM

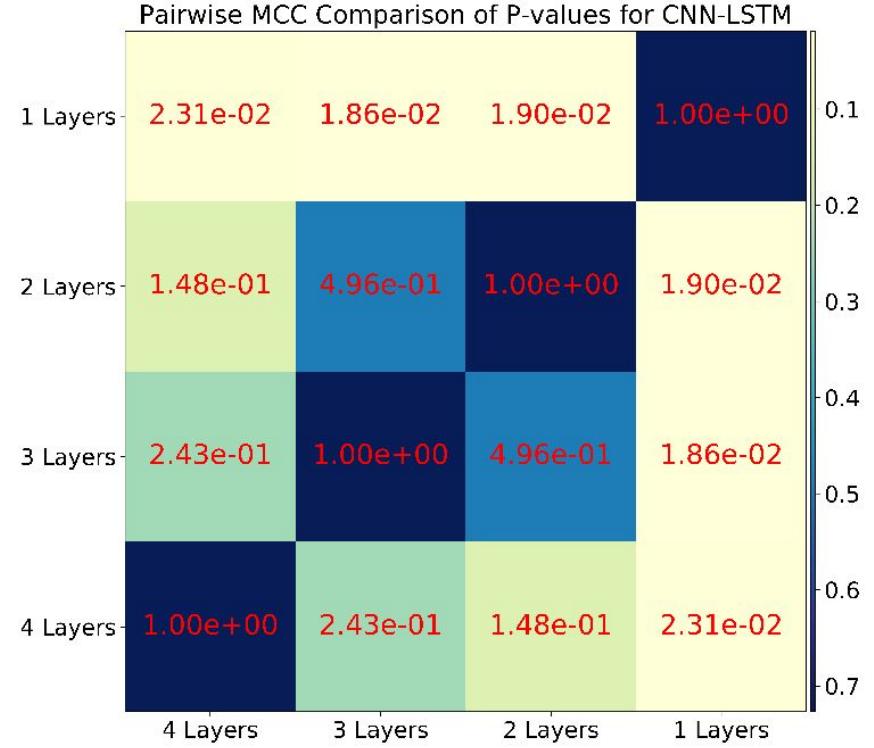


Figure S2. The $p$-values of pairwise model depth comparison are calculated by using the Wilcoxon signed-rank test.
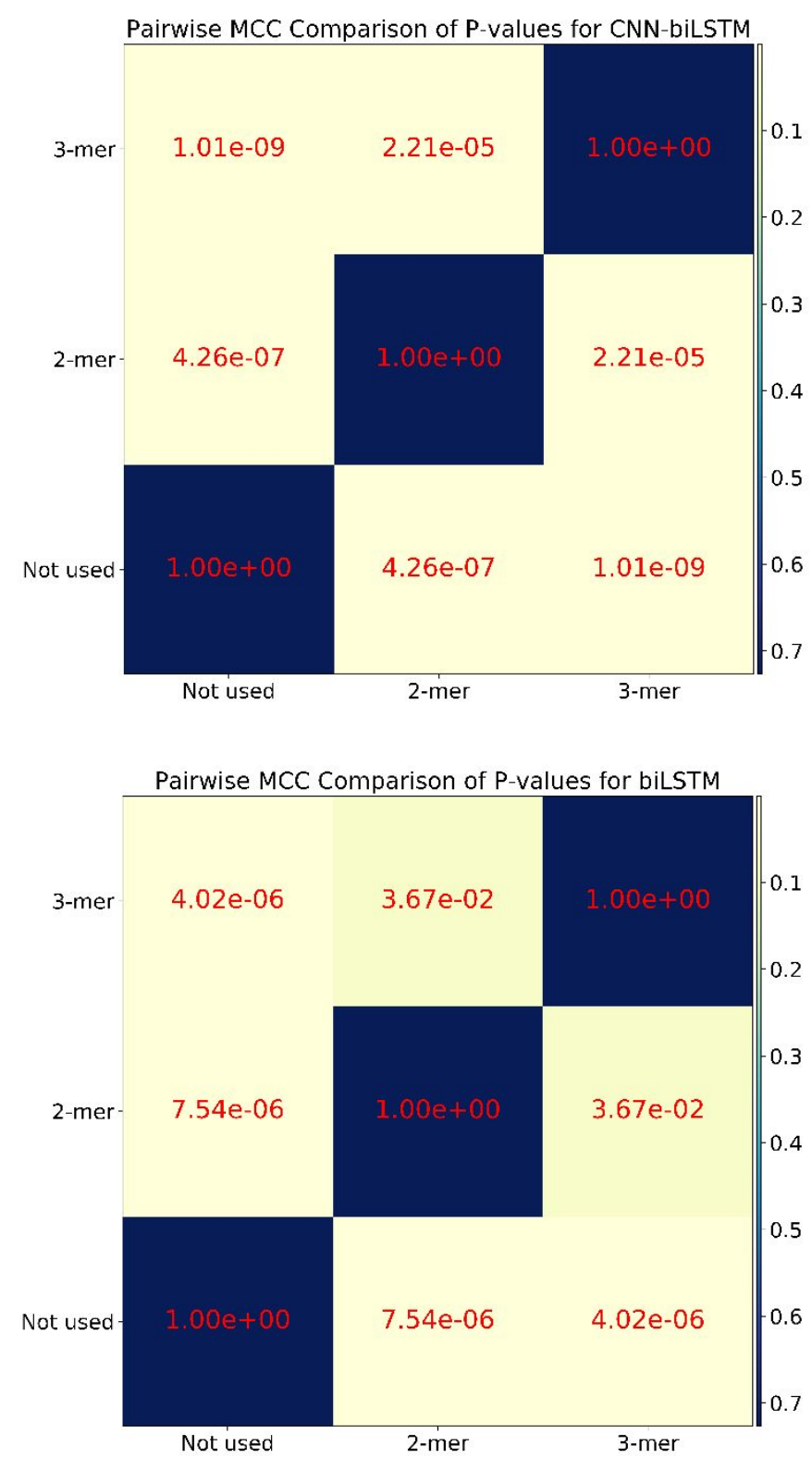

Figure S3. The $p$-values of pairwise $k$-mer comparison are calculated by using the Wilcoxon signed-rank test. 

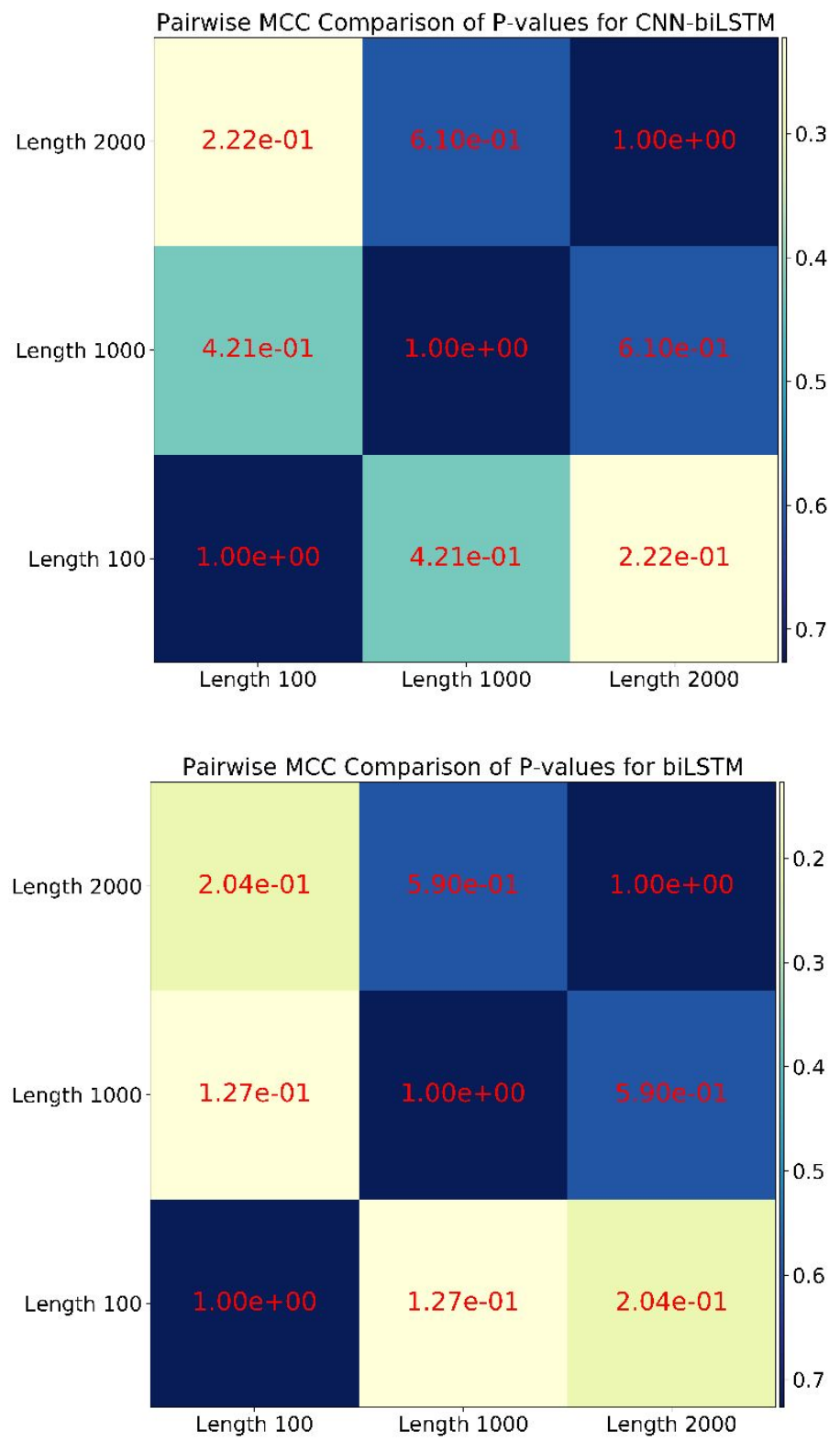

Figure S4. The $p$-values of pairwise input sequence lengths comparison are calculated by using the Wilcoxon signed-rank test. 
A

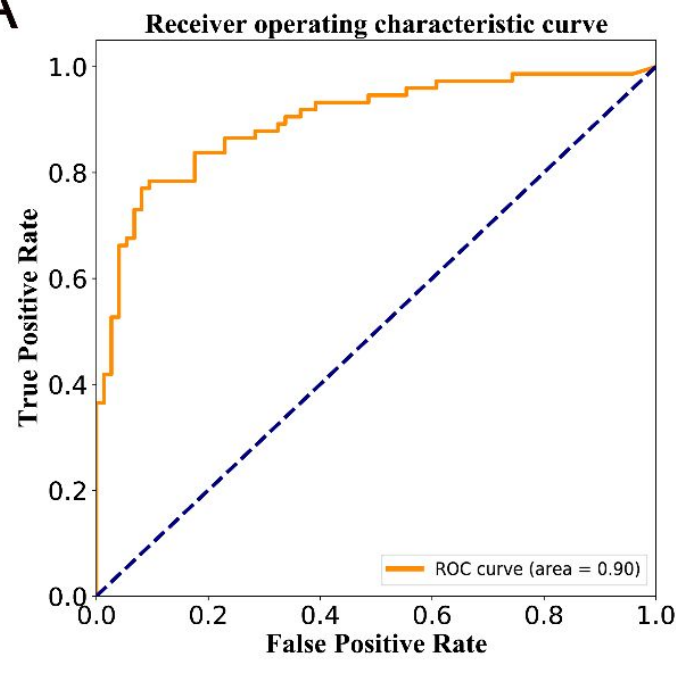

B

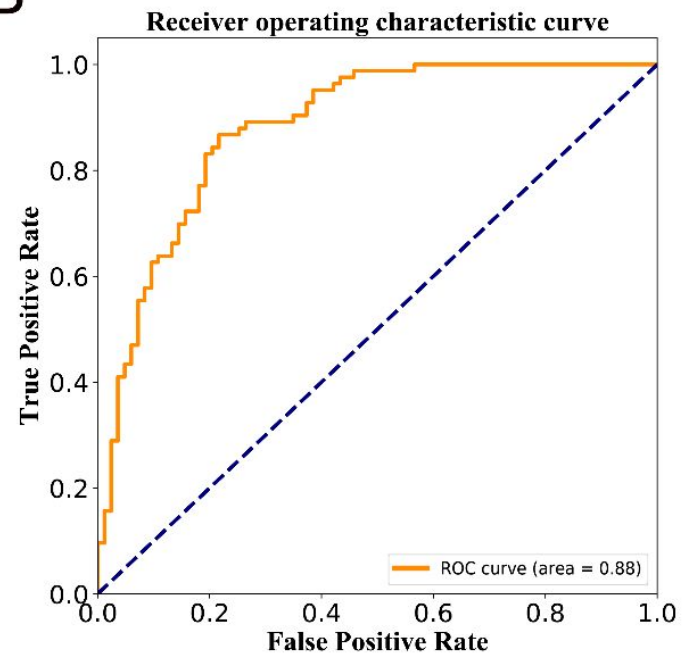

C

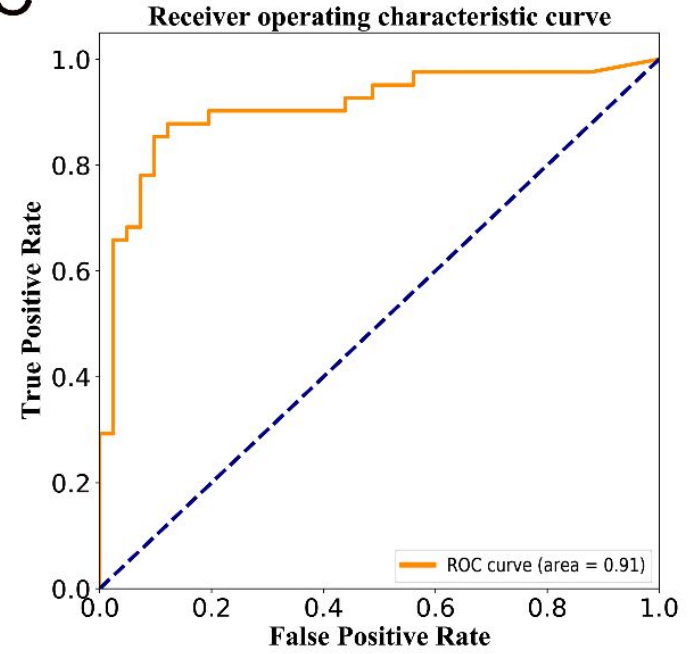

Precision recall curve

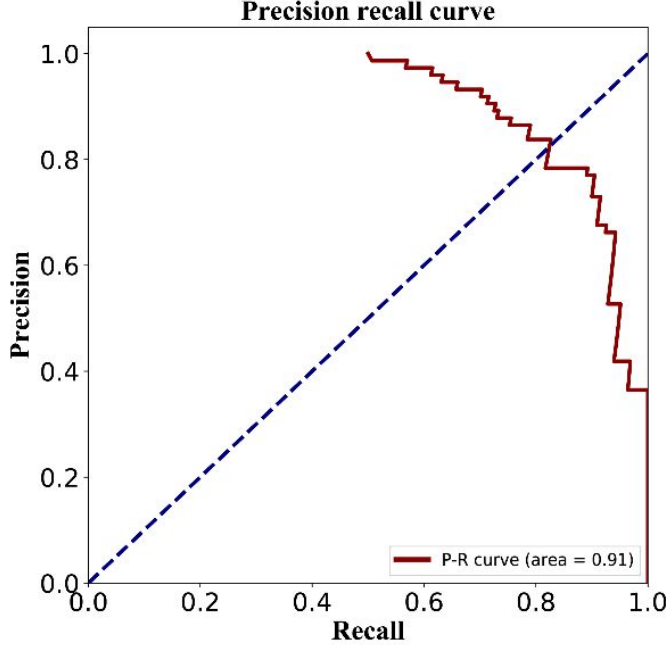

Precision recall curve

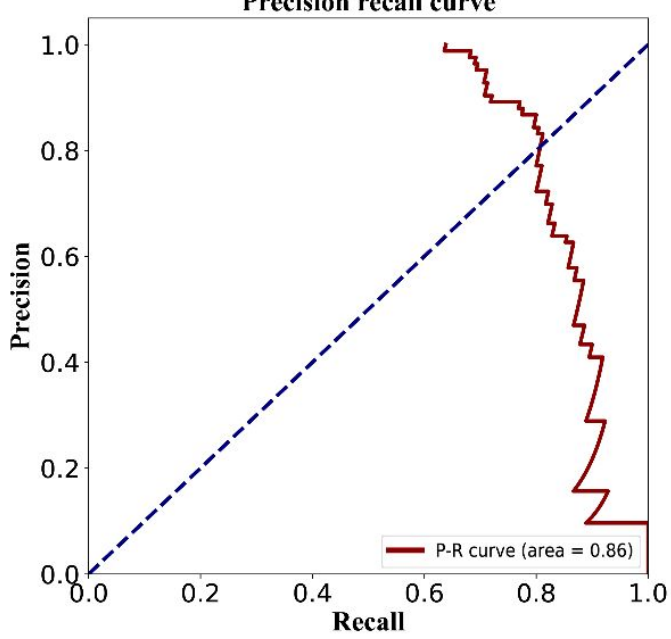

Precision recall curve

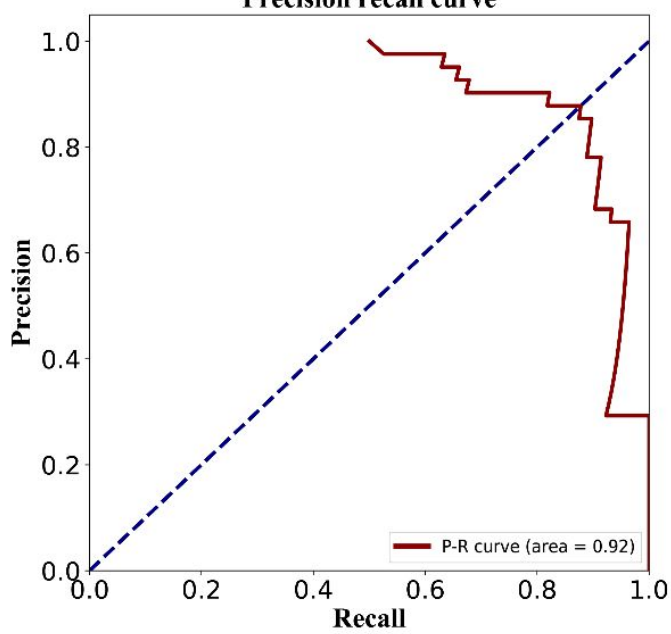

Figure S5. ROC and PR curves are shown for the best encoding ways trained on: A Doench dataset. B Wang/Xu dataset. C Moreno-Mateos dataset. 


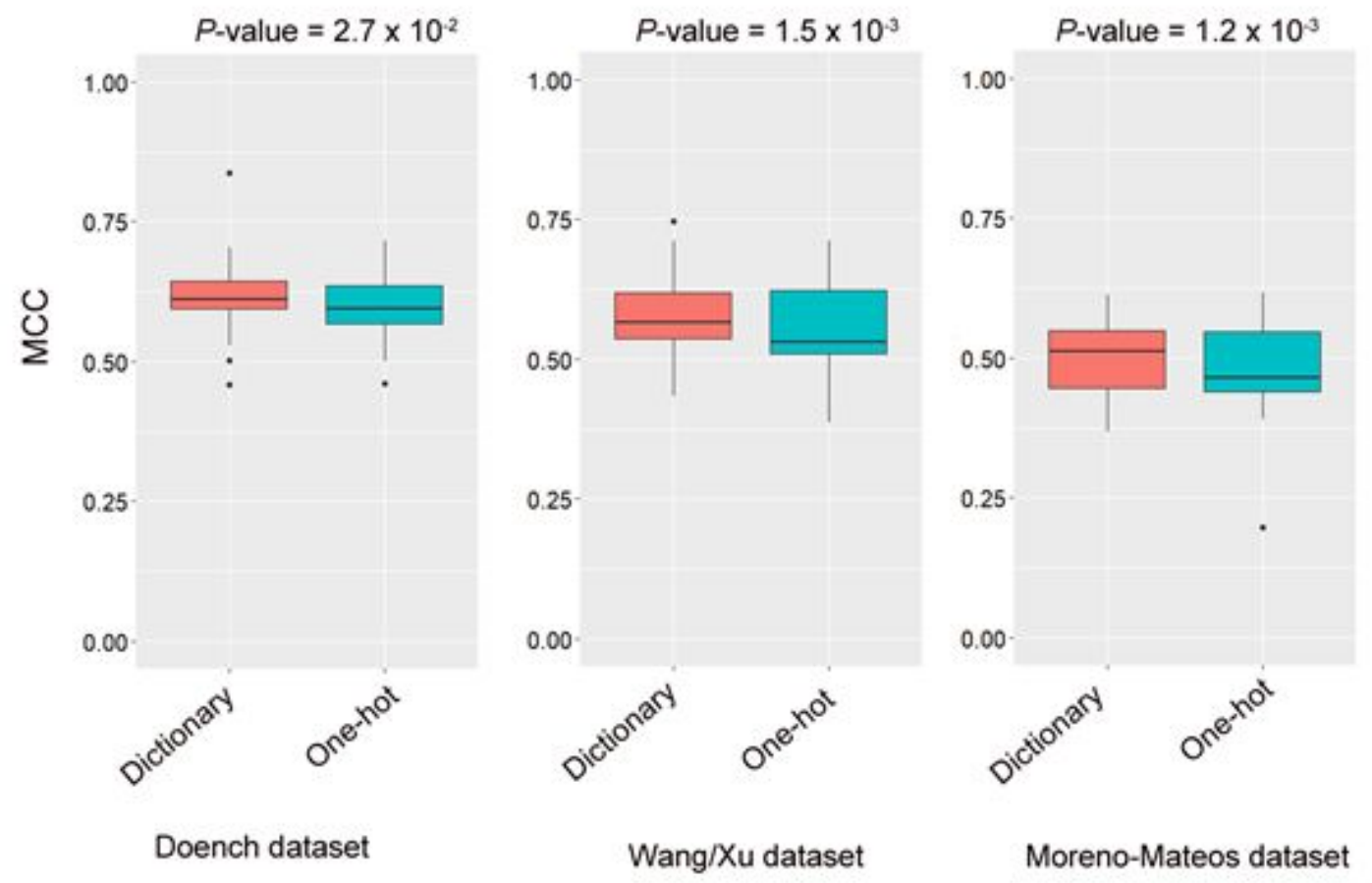

Figure S6. The distribution of MCC scores for two encoding ways including $k$-mer-based algorithms on three datasets. The $p$-values of pairwise encoding comparison are calculated by using the Wilcoxon signed-rank test. 


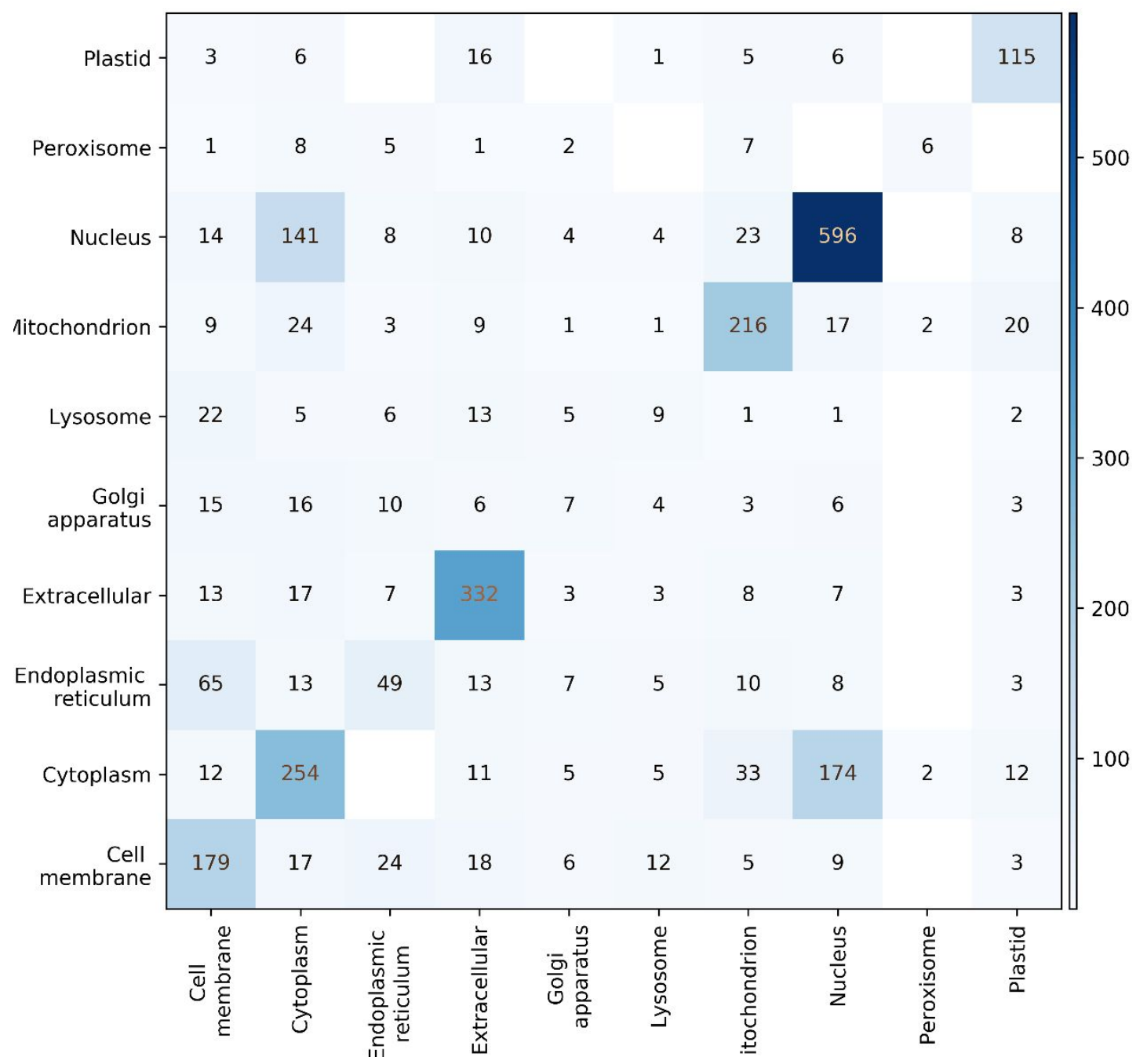

Figure S7. The confusion matrix of subcellular localization classification. 


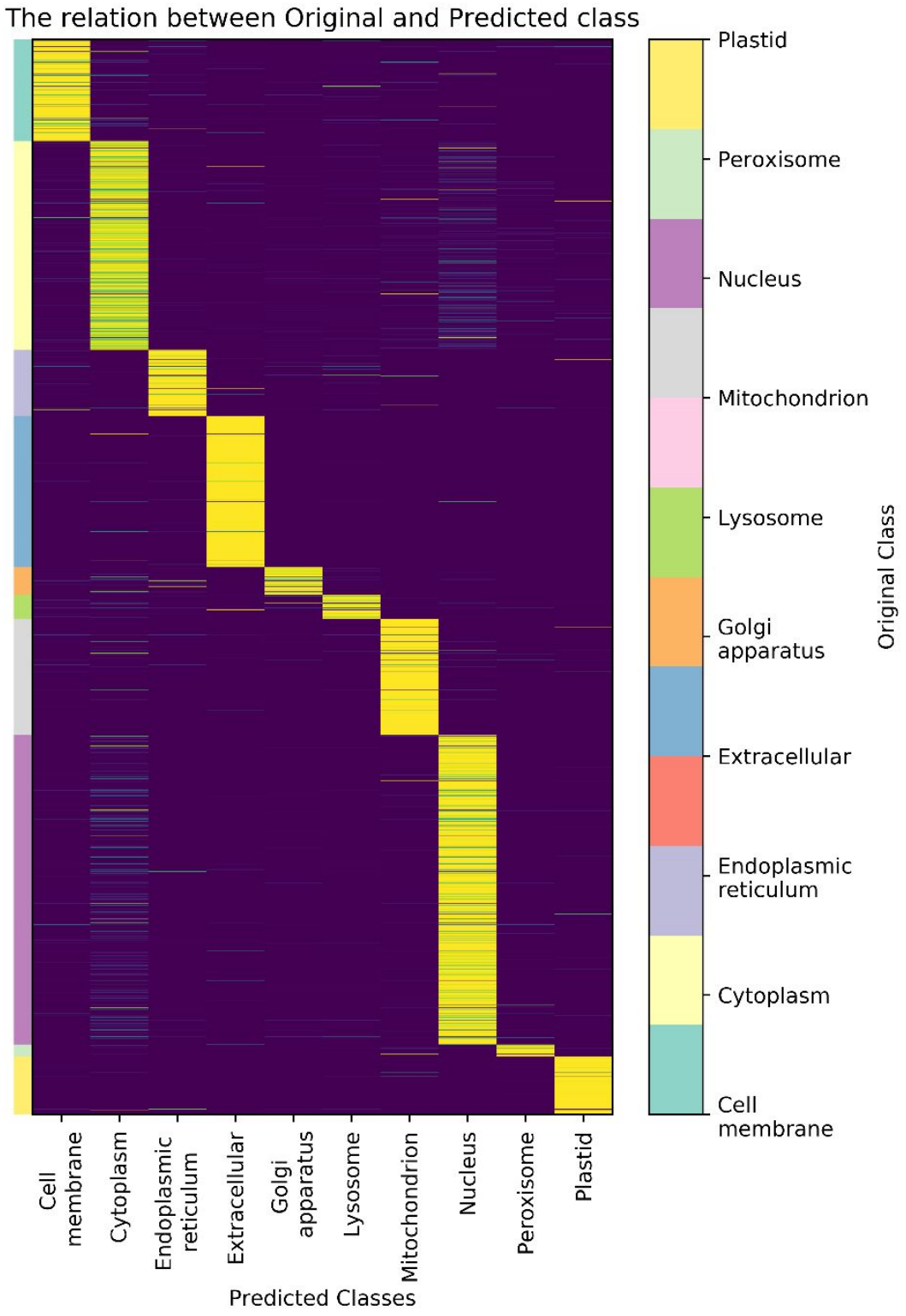

Figure S8. The self-attention plotting of subcellular localization classification. 